\title{
TITLE:
}

\section{In vitro regeneration of kidney from pluripotent stem cells.}

$\operatorname{AUTHOR}(S)$ :

Osafune, Kenji

CITATION:

Osafune, Kenji. In vitro regeneration of kidney from pluripotent stem cells.. Experimental cell research 2010, 316(16): 2571-2577

ISSUE DATE:

2010-10-01

URL:

http://hdl.handle.net/2433/128879

\section{RIGHT:}

(c) 2010 Elsevier Inc.; This is not the published version. Please cite only the published version.; この論文は出版社版でありません。引用の際に は出版社版をご確認ご利用ください。 
In vitro regeneration of kidney from pluripotent stem cells

\section{Authors' names and affiliations}

Kenji Osafune

${ }^{1}$ Center for iPS Cell Research and Application (CiRA), Kyoto University, 53

Kawahara-cho, Shogoin, Sakyo-ku, Kyoto 606-8507, Japan, ${ }^{2}$ PRESTO, ${ }^{3}$ JST Yamanaka iPS Cell Special Project, Japan Science and Technology Agency (JST), 4-1-8 Honcho,

Kawaguchi, Saitama 332-0012, Japan.

Email: osafu@cira.kyoto-u.ac.jp

Phone: +81-75-366-7058, Fax: +81-75-366-7077

\section{Running title}

Kidney regeneration from stem cells

\section{Abstract}

Although renal transplantation has proved a successful treatment for the patients with end-stage renal failure, the therapy is hampered by the problem of serious shortage of donor organs. Regenerative medicine using stem cells, including cell transplantation therapy, needs to be developed to solve the problem. We previously identified the multipotent progenitor cells in the embryonic mouse kidney that can give rise to several kinds of epithelial cells found in adult kidney, such as glomerular podocytes and renal 
tubular epithelia. Establishing the method to generate the progenitors from human pluripotent stem cells that have the capacity to indefinitely proliferate in vitro is required for the development of a kidney regeneration strategy. We review the current status of the research on the differentiation of pluripotent stem cells into renal lineages and describe cues to promote this research field.

\section{Key words}

Kidney regeneration, Pluripotent stem cell, iPS cells, ES cells, Animal cap, Kidney development

\section{Text}

\section{Introduction}

An increasing number of patients with end-stage renal failure undergo dialysis therapy around the world. It causes both medical and medico economical problems. Renal transplantation has proved a successful therapy for most patients with end-stage renal failure, as the therapy results in a significant improvement in the patient's quality of life, and prolongs survival. And it is also considered cost-effective [1]. However, the annual increase in the number of new patients with end-stage renal disease who need a renal transplant and the widening gap between the demand for and the supply of donor kidney, has led to a progressive shortage of donor organs for transplant around the world. This 
has become a serious issue and is even worsened by limited graft survival due to immune rejection [1].

Among strategies to overcome these problems is kidney regeneration using stem cells. Stem cells contain two large categories; organ-specific or somatic stem cells and pluripotent stem cells. In contrast to organ-specific stem cells that generally have the limited potential for growth and differentiation, pluripotent stem cells, such as embryonic stem (ES) cells [2-4] and induced pluripotent stem (iPS) cells [5-7], have a virtually unlimited replicative capacity on culture dishes and are theoretically able to give rise to any cell types in the body. The cell types have increasingly been used as a model system for understanding developmental mechanisms. In addition, in vitro culture and the differentiation of the stem cells offer unique opportunities for disease modeling, drug discovery, toxicology and cell replacement therapy [8]. The generation of specific functional cell types from ES cells has been demonstrated, including neuron, vascular endothelia, cardiomyocytes, hematopoietic cells, pancreatic insulin-producing cells, and hepatocyte-like cells [9]. However, the protocol for in vitro differentiation of pluripotent stem cells into renal lineage cells has not been fully established.

Other approaches to regenerate kidney have also been investigated using organ-specific local stem cells within the kidney and bone marrow-derived 
hematopoietic stem cells [10]. Kidney regeneration using mesenchymal stem cells localized in bone marrow has also been investigated [11].

This review first summarizes the mechanisms of kidney development, then focuses on the directed differentiation of pluripotent stem cells into renal lineages based on the knowledge of kidney development, and finally describes potential applications of this strategy for various research fields. In vitro generation of kidney using the undifferentiated cell mass in amphibian eggs, similar to mammalian pluripotent stem cells in that the cell mass can differentiate into various organs in vitro, is also described as references to kidney regeneration in mammals.

\section{Mechanisms of kidney development}

Vertebrates develop successively three kidneys; pronephros, mesonephros and metanephros. The three kidneys consist of a basic functional unit, the nephron, although the number of nephron differs among the kidneys [12]. The kidneys are derived from a portion of the early embryonic germ layer, intermediate mesoderm that is located between lateral and paraxial mesoderms [13]. Lineage tracing experiments have recently demonstrated that intermediate mesodermal cells expressing Odd-skipped related (Osr) -1, an essential transcriptional factor for intermediate mesoderm and kidney development, give rise to all components consisting of mammalian adult kidney 
metanephros [13]. Metanephros is formed by reciprocal interaction between two tissues derived from intermediate mesoderm - metanephric mesenchyme and ureteric bud [12]. The ureteric bud induces the metanephric mesenchyme to differentiate into the epithelia of glomeruli and renal tubules. By creating a novel culture system which uses the co-culture with the cell line expressing a renal-epithelializing factor Wnt4, we previously demonstrated that mouse metanephric mesenchyme contains multipotent progenitor cells that can give rise to several kinds of epithelial cells found in adult kidney, such as glomerular podocytes and epithelia of proximal and distal renal tubules and Henle's loop [14] (Figure 1A, B). The progenitor cells are contained only in the cell population strongly expressing Sall1, a zinc finger transcriptional factor that is essential for kidney development. It was also shown that these progenitor cells can reconstitute a three dimensional kidney structure in vitro, which contains glomeruli- and tubule-like components in an organ culture setting (Figure 1C, D). The presence of the progenitor cells was also demonstrated in vivo by using a lineage tracing experiment for Six2, which is also an essential transcriptional factor for kidney development [15].

The progenitors in metanephric mesenchyme differentiate into epithelia after Wnt stimulation, but a cell fate decision is required for further differentiation toward glomerular podocytes, proximal or distal renal tubules, or Henle's loop. Molecular 
mechanisms of the cell fate decision is largely unknown, although it was demonstrated that Notch2 is required for the differentiation of proximal nephron structures, such as podocytes and proximal renal tubules, as Notch2 deletion leads to the impaired formation of these proximal structures [16]. There may be many other molecules involved in the lineage commitment, and they remain to be elucidated. Metanephric mesenchyme may well contain at least two other progenitor populations in addition to the epithelial progenitors; 1) vascular progenitor cells that can give rise to vascular and glomerular endothelial cells, vascular smooth muscle cells (pericytes) and mesangial cells, and 2) stromal progenitors eventually developing into interstitial cells within adult kidney. The ureteric bud is known to elaborate the lower urinary tract system - from collecting ducts through renal pelvis and ureters to a part of the urinary bladder [12]. Most of the mechanisms of the lineage commitment, by which the intermediate mesoderm gives rise to ureteric bud, stromal and vascular cells, are unknown. Further examinations will be required to elucidate the mechanisms, which will eventually help us completely understand the commitment of multiple cell lineages within a kidney and in reproduce it in vitro from pluripotent stem cells.

\section{Animal cap in fertilized eggs of amphibians}


Amphibians have been used as experimental animals for the research of developmental biology mainly because of its easiness to handle and observe. In amphibian eggs, the ectodermal cell mass of mid-blastula embryos, called animal cap, is similar to ES and iPS cells in mammals in that it has a potential of multipotent differentiation. The animal cap can be easily excised from fertilized eggs and cultured as explants in vitro in simple saline solution. After the treatment with differentiation-inducing factors, animal cap can differentiate into various tissues and organs in vitro [17]. The pronephros is a simple excretory organ of Xenopus larvae with only one nephron, consisting of glomus, a filtering unit equivalent to glomerulus in metanephros, tubule and duct. When animal caps are treated with the combination of activin A, a protein inducer belonging to transforming growth factor (TGF) - $\beta$ supperfamily, and retinoic acid, the differentiation into pronephric tubules was observed [18]. In the same differentiation system, the marker gene expression for pronephric glomus was detected [19]. We demonstrated that pronephric duct, a third component of pronephric kidney, can be generated in the explants, in addition to pronephric tubule and glomus, and that the pronephros formed in vitro is similar to that in Xenopus embryos both histologically and in gene expression (Figure 2) [20]. The pronephic tubule and duct also have the similar ultrastructure to those of Xenopus embryos by the examination with electron microscopy. 
The pronephros formed from animal cap in vitro shows temporarily a similar gene expression pattern to that in Xenopus embryos. Using the in vitro system to generate pronephric tissue, the molecular mechanisms underlying pronephros development has been investigated and some molecules involved in the formation of pronephros were identified [17]. Although the pronephros produced in vitro from animal cap cannot be directly translated to clinical application, this system should facilitate the study of kidney regeneration and may promote a shift from tissue engineering to clinical applications. Indeed, this induction method established for animal cap using activin $\mathrm{A}$ and retinoic acid has been applied for a differentiation experiment from mouse ES cells into renal lineages [21].

\section{ES cells}

ES cells are pluripotent stem cells derived from the inner cell mass of fertilized eggs in mammals. The first derivation of mouse ES cells by Evans and Martin was described in 1981 [2, 3] and human ES cells was established in about 20 years later [4]. Both mouse and human ES cells have a virtually unlimited replicative capacity and are theoretically able to give rise to any cell types in the body. Since its derivation, mouse ES cells have mainly been used as a research tool to generate experimental mouse models by the combination with gene targeting techniques. However, after the success in human ES 
cell derivation, regenerative medicine strategy has been considered closer to clinical applications, which aim at accomplishing the functional recovery of dysfunctional organs by transplanting organ-specific cell types generated from ES cells in vitro. The research on the in vitro differentiation of ES cells into specific cell types of various organs has since been more vigorously investigated to develop cell transplantation therapies.

In vivo injection of ES cells into immunocompromized mice can produce teratoma, which are tumors containing cells of all three embryonic germ layers (ectoderm, mesoderm and endoderm). It was shown that glomerulus- and tubule-like structures can be formed in teratomas derived from mouse [22] and human ES cells [4], which demonstrate that ES cells do have a potential to differentiate into kidney lineages. Furthermore, fundamental research aiming to establish the methods to efficiently induce mouse ES cells into renal progenitors and fully differentiated renal cells has already been carried out [21, 23-26]. Mouse ES cells treated with the combination of hepatocyte growth factor (HGF) and activin $\mathrm{A}$ in addition to the transfection of renal-epithelializing factor Wnt4 [23], the combination of activin A, Bone morphogenetic protein (BMP)-7 and retinoic acid [21], activin A alone [24], BMP-4 alone [25], or the combination of four chemical compounds [26], produced cells expressing markers for intermediate 
mesoderm, developing kidney and fully differentiated renal cells. In some reports, the induced cells formed tubule-like structures in or were incorporated into developing mouse kidneys [21, 23, 24]. Few reports have described the directed differentiation of human ES cells into renal lineage. It was shown that human ES cells differentiated in vitro into cells expressing WT1 and rennin, marker genes for glomeruli, following the treatment with growth factors [27]. Recently it has been reported that the combinational treatment with activin $\mathrm{A}$, retinoic acid, and BMP-4 or BMP-7 also induces marker gene expression for intermediate mesoderm and developing kidney from human ES cells [28].

In summary, these data suggest that ES cells are a potential source for kidney regeneration, although the efficiency or the induction rate of generation of renal cells from ES cells is unknown in many cases. It remains to be determined whether kidney lineage cells produced from ES cells have a function as kidney. It is also unknown whether tubule-like structures or cells integrated into kidney are metanephric or of earlier kidneys (pronephros and mesonephros). The research on kidney regeneration using ES cells is at its beginning, and further examinations are needed to develop this research field.

\section{iPS cells}


One of the more remarkable observations in the past century is that the differentiation is not a unidirectional process. Instead, we can turn back the clock on differentiated cells. This rewinding of the developmental clock is termed nuclear reprogramming and is often defined as the process whereby an adult somatic nucleus has developmental potential restored to it. There are two conventional ways that nuclear reprogramming could be accomplished: somatic cell nuclear transfer or cloning and cell fusion [29]. Somatic cell nuclear transfer (SCNT), a procedure in which the nucleus of an adult cell is injected into an unfertilized egg whose chromosomes have been removed, has demonstrated that the genome of adult cells can be reset to an embryonic state. Using this strategy researchers have generated cloned embryos that possess the potential to develop into an adult animal or become an ES cell line that is genetically identical to that of the donor nucleus. These experiments demonstrated that no irreversible changes are made to the genome during development and further showed that animal oocytes harbored factors that could accomplish nuclear reprogramming. In a related series of experiments, a number of researchers have shown that when somatic cells are fused with ES cells, the resulting tetraploid hybrid cells silence the expression of somatic genes and establish a program of transcription indistinguishable from ES cells, indicating that ES cells contain the necessary reprogramming activities to accomplish 
this transformation. Thus, the cytoplasm of the enucleated oocyte and the ES cell is able to re-establish the pluripotent state via a mechanism dependent on global epigenetic and transcriptional changes. The mechanism by which this transformation from adult somatic cells to pluripotent stem cells occurred and the mediators of nuclear reprogramming was largely undefined.

In a breakthrough experiment, Takahashi and Yamanaka identified four factors normally found in ES cells - Oct3/4, Sox2, c-Myc, and Klf4 - and reported that they were sufficient to reprogram both mouse and human somatic cells to closely resemble mouse and human ES cells [5-7]. They called the cell type induced pluripotent stem (iPS) cells. Since the reports, the technique has been rapidly confirmed, improved and subsequently applied to successfully reprogram somatic cells.

Therapeutic approaches using human ES cells face the two major problems. One is the ethical issue derived from the usage of human fertilized eggs, and the other is immune rejection in any cell or tissue transplantation due to histocompatibility antigenic differences between ES cells and patients. These problems have become resolvable by using iPS cells, which can be generated from somatic cells of patients. In the next step after iPS cell derivation, significant progress has been made in re-differentiating iPS cells into somatic cells including active motor neurons [30], insulin-secreting islet-like 
clusters [31], and a number of cardiovascular cells (arterial endothelium, venous endothelium, lymphatic endothelium, cardiomyocytes) [32, 33]. To date, few reports have described the generation of renal lineage cells from iPS cells. However, the phenotype of iPS cells is indistinguishable from ES cells and accumulated experiences and knowledge using ES cells to generate mesodermal and renal lineage cells are potentially applicable to iPS cell differentiation culture.

The iPS cell technology enables the creation of patient-specific pluripotent cell lines that carry disease genotypes. The generation of disease-specific iPS lines has been first reported from a familial form of a neurodegenerative disorder, amyotrophic lateral sclerosis (ALS) [34]. Disease-specific iPS cells has also been produced by other groups from more than 10 different genetic diseases, including Parkinson's disease, type-1 diabetes, Duchenne's and Becker's muscular dystrophy, adenosine deaminase deficiency-related severe combined immunodeficiency, Shwachman-Bodian-Diamond syndrome, Gaucher disease type III, Huntington's disease, Down's syndrome, and the carrier state of Lesch-Nyhan syndrome [35]. These cell lines could be used both as in vitro models for the study of disease and as potential sources of material for cell replacement therapy. Ultimately, a greater understanding of the process by which cellular identity is shaped and altered may allow for the generation of particular cell 
types for the treatment of degenerative diseases. These approaches can be also applied to genetic renal diseases.

Other clinical applications using ES or iPS cell technology include the usage of stem cell-derived tissues for in vitro toxicology screening. For example, it has been shown that human ES or iPS cell-derived cardiomyocytes can be used to examine toxic effects of drug compounds on caridomyocytes, such as drug-induced QT interval prolongation that can lead to sudden cardiac death and is a major safety concern for the drug industry [36]. The similar approaches may well be the use of ES or iPS cell-derived hepatocytes and renal cells in testing hepatotoxicity and nephrotoxicity of drug compounds.

\section{Cues to develop kidney regeneration strategy from pluripotent stem cells}

By mimicking signals used in embryonic development, to the extent that they are known, a stepwise protocol was explored to differentiate ES or iPS cells into some specific cell types in adult organs, such as pancreas and liver [37, 38]. For the generation of insulin-producing cells in pancreas, ES cells has been first differentiated into definitive endoderm, then foregut endoderm, followed by pancreatic progenitors, and eventually insulin-expressing endocrine cells [37]. These stepwise approaches may be used in generating renal lineage cells from ES or iPS cells, and it should involve 
directing the pluripotent stem cells first to form intermediate mesoderm, then the renal progenitors, followed by the eventual formation of functional renal cells found in adult kidney.

It has recently been shown that significant differences exist in the differentiation potential of different human ES cell lines, even though the observable differences in the pluripotent state are marginal [39]. Moreover, some human ES cell lines have a propensity to differentiate into certain lineages or cell types. These results also suggested that pluripotent stem cells derived by other means including iPS cells may well show a similar variability. The finding also underscores the importance of using suitable stem cell lines for the renal lineage differentiation.

Directed differentiation of pluripotent stem cells into specific organ lineages is usually carried out through the combination of cell-cell interactions (co-culture with some cell lines), exposure to diffusible factors (growth factor) and other positional cues (extracellular matrix) that ultimately alter gene expression conferring a specific cellular identity and function. Direct genetic manipulations on stem cells using the overexpression of cDNA, small interfering RNA (siRNA) or short hairpin RNA (shRNA) has been also performed to generate specific cell types. It has been recently demonstrated that high-throughput screening of chemical compounds can be used to 
identify a small molecule that has a potential to induce the differentiation of pluripotent stem cells into specific lineages [40]. In the report, a chemical compound, (-) indolactam $\mathrm{V}$, known to activates protein kinase $\mathrm{C}$ (PKC) signaling, was shown to induces the differentiation of a substantial number of pancreatic progenitor cells from human ES cells. It was also shown that the activated PKC signal is involved in the pancreatic specification in human ES cell culture [40]. Similarly, chemical screens in an unbiased high-throughput method can be applied for the identification of chemical compounds to induce renal lineage differentiation from pluripotent stem cells and the elucidation of mechanisms of kidney differentiation.

\section{Conclusion}

Research for the kidney regeneration strategy using pluripotent stem cells is just at its beginning. Of course, continued efforts to elucidate the developmental mechanisms of mesoderm formation, the specification into kidney lineages, and nephron specification should be made. These investigations are necessary for the establishment of differentiation protocols from pluripotent stem cells into renal lineages. Further investigations using pluripotent stem cells as a model for kidney development will facilitate our understanding of kidney precursor specification and the development of new cell-based therapies for the treatment of renal diseases. Because of the annual 
increase in the number of patients with end-stage renal failure who have to undergo dialysis therapy around the world, regenerative medicine strategies using pluripotent stem cells needs to be developed.

\section{Acknowledgement}

The author would like to thank members of CiRA, Kyoto University, for scientific support and helpful discussions and Akemi Nakamura for editing the manuscript.

\section{References}

[1] B. M. Shrestha, Strategies for reducing the renal transplant waiting list: a review, Exp. Clin. Transplant. 7 (2009) 173-179.

[2] M. J. Evans, M. H. Kaufman, Establishment in culture of pluripotential cells from mouse embryos, Nature 292 (1981) 154-156.

[3] G. R. Martin, Isolation of a pluripotent cell line from early mouse embryos cultured in medium conditioned by teratocarcinoma stem cells, Proc. Natl. Acad. Sci. USA 78 (1981) 7634-7638.

[4] J. A. Thomson, J. Itskovitz-Eldor, S. S. Shapiro, M. A. Waknitz, J. J. Swiergiel, V. S. Marshall, J. M. Jones, Embryonic stem cell lines derived from human blastocysts, Science 282 (1998) 1145-1147. 
[5] K. Takahashi, S. Yamanaka, Induction of pluripotent stem cells from mouse embryonic and adult fibroblast cultures by defined factors, Cell 126 (2006) 663-676.

[6] K. Takahashi, K. Tanabe, M. Ohnuki, M. Narita, T. Ichisaka, K. Tomoda, S. Yamanaka, Induction of pluripotent stem cells from adult human fibroblasts by defined factors, Cell 131 (2007) 861-872.

[7] J. Yu, M. A. Vodyanik, K. Smuga-Otto, J. Antosiewicz-Bourget, J. L. Frane, S. Tian, J. Nie, G. A. Jonsdottir, V. Ruotti, R. Stewart, I. I. Slukvin, J. A. Thomson, Induced pluripotent stem cell lines derived from human somatic cells, Science 318 (2007) 1917-1920.

[8] P. H. Lerou, G. Q. Daley, Therapeutic potential of embryonic stem cells, Blood Rev. 19 (2005) 321-331.

[9] C. E. Murry, G. Keller, Differentiation of embryonic stem cells to clinically relevant populations: lessons from embryonic development, Cell 132 (2008) 661-680.

[10] C. Roufosse, H. T. Cook, Stem cells and renal regeneration, Nephron Exp. Nephrol. 109 (2008) e39-45.

[11] T. Yokoo, A. Fukui, K. Matsumoto, T. Ohashi, Y. Sado, H. Suzuki, T. Kawamura, M. Okabe, T. Hosoya, E. Kobayashi, Generation of a transplantable 
erythropoietin-producer derived from human mesenchymal stem cells, Transplantation 85 (2008) 1654-1658.

[12] L. Saxen, Organogenesis of the kidney, Cambridge: Cambridge University Press (1987).

[13] J. W. Mugford, P. Sipilä, J. A. McMahon, A. P. McMahon, Osr1 expression demarcates a multi-potent population of intermediate mesoderm that undergoes progressive restriction to an Osr1-dependent nephron progenitor compartment within the mammalian kidney, Dev. Biol. 324 (2008) 88-98.

[14] K. Osafune, M. Takasato, A. Kispert, M. Asashima, R. Nishinakamura, Identification of multipotent progenitors in the embryonic mouse kidney by a novel colony-forming assay, Development 133 (2006) 151-161.

[15] A. Kobayashi, M. T. Valerius, J. W. Mugford, T. J. Carroll, M. Self, G. Oliver, A. P. McMahon, Six2 defines and regulates a multipotent self-renewing nephron progenitor population throughout mammalian kidney development, Cell Stem Cell 3 (2008) 169-181.

[16] H. T. Cheng, M. Kim, M. T. Valerius, K. Surendran, K. Schuster-Gossler, A. Gossler, A. P. McMahon, R. Kopan, Notch2, but not Notch1, is required for proximal fate acquisition in the mammalian nephron, Development 134 (2007) 801-811. 
[17] M. Asashima, Y. Ito, T. Chan, T. Michiue, M. Nakanishi, K. Suzuki, K. Hitachi, K.

Okabayashi, A. Kondow, T. Ariizumi, In vitro organogenesis from undifferentiated cells in Xenopus, Dev. Dyn. 238 (2009) 1309-1320.

[18] N. Moriya, H. Uchiyama, M. Asashima, Induction of pronephric tubules by activin and retinoic acid in presumptive ectoderm of Xenopus laevis, Dev. Growth Differ. 35 (1993) 123-128.

[19] H. C. Brennan, S. Nijjar, E. A. Jones, The specification and growth factor inducibility of the pronephric glomus in Xenopus laevis, Development 126 (1999) $5847-5856$.

[20] K. Osafune, R. Nishinakamura, S. Komazaki, M. Asashima, In vitro induction of the pronephric duct in Xenopus explants, Dev. Growth Differ. 44 (2002) 161-167.

[21] D. Kim, G. R. Dressler, Nephrogenic factors promote differentiation of mouse embryonic stem cells into renal epithelia, J. Am. Soc. Nephrol. 16 (2005) 3527-3534.

[22] M. Yamamoto, L. Cui, K. Johkura, K. Asanuma, Y. Okouchi, N. Ogiwara, K. Sasaki, Branching ducts similar to mesonephric ducts or ureteric buds in teratomas originating from mouse embryonic stem cells, Am. J. Physiol. Renal Physiol. 290 (2006) F52-F60. 
[23] T. Kobayashi, H. Tanaka, H. Kuwana, S. Inoshita, H. Teraoka, S. Sasaki, Y. Terada, Wnt4-transformed mouse embryonic stem cells differentiate into renal tubular cells, Biochem. Biophys. Res. Commun. 336 (2005) 3527-3534.

[24] C. Vigneau, K. Polgar, G. Striker, J. Elliott, D. Hyink, O. Weber, H. J. Fehling, G. Keller, C. Burrow, P. Wilson, Mouse embryonic stem cell-derived embryoid bodies generate progenitors that integrate long term into renal proximal tubules in vivo, J. Am. Soc. Nephrol. 18 (2007) 1709-1720.

[25] S. J. Bruce, R. W. Rea, A. L. Steptoe, M. Busslinger, J. F. Bertram, A. C. Perkins, In vitro differentiation of murine embryonic stem cells toward a renal lineage, Differentiation 75 (2007) 337-349.

[26] S. Mae, S. Shirasawa, S. Yoshie, F. Sato, Y. Kanoh, H. Ichikawa, T. Yokoyama, F. Yue, D. Tomotsune, K. Sasaki, Combination of small molecules enhances differentiation of mouse embryonic stem cells into intermediate mesoderm through BMP7-positive cells, Biochem. Biophys. Res. Commun. 393 (2010) 877-882.

[27] M. Schuldiner, O. Yanuka, J. Itskovitz-Eldor, D. A. Melton, N. Benvenisty, Effects of eight growth factors on the differentiation of cells derived from human embryonic stem cells, Proc. Natl. Acad. Sci. USA 97 (2000) 11307-11312. 
[28] C. A. Batchelder, C. I. Lee, D. G. Matsell, M. C. Yoder, A. F. Tarantal, Renal ontogeny in the rhesus monkey (Macaca mulatta) and directed differentiation of human embryonic stem cells towards kidney precursors, Differentiation 78 (2009) 45-56.

[29] S. Yamanaka, Pluripotency and nuclear reprogramming, Philos. Trans. R. Soc. Lond. B. Biol. Sci. 363 (2008) 2079-2087.

[30] S. Karumbayaram, B. G. Novitch, M. Patterson, J. A. Umbach, L. Richter, A. Lindgren, A. E. Conway, A. T. Clark, S. A. Goldman, K. Plath, M. Wiedau-Pazos, H. I. Kornblum, W. E. Lowry, Directed differentiation of human-induced pluripotent stem cells generates active motor neurons, Stem Cells 27 (2009) 806-811.

[31] K. Tateishi, J. He, O. Taranova, G. Liang, A. C. D’Alessio, Y. Zhang, Generation of insulin-secreting islet-like clusters from human skin fibroblasts, J. Biol. Chem. 283 (2008) 31601-31607.

[32] D. Taura, M. Sone, K. Homma, N. Oyamada, K. Takahashi, N. Tamura, S. Yamanaka, K. Nakao, Induction and isolation of vascular cells from human induced pluripotent stem cells--brief report, Arterioscler. Thromb. Vasc. Biol. 29 (2009) 1100-1103. 
[33] G. Narazaki, H. Uosaki, M. Teranishi, K. Okita, B. Kim, S. Matsuoka, S. Yamanaka, J. K. Yamashita, Directed and systematic differentiation of cardiovascular cells from mouse induced pluripotent stem cells, Circulation 118 (2008) 498-506.

[34] J. T. Dimos, K. T. Rodolfa, K. K. Niakan, L. M. Weisenthal, H. Mitsumoto, W. Chung, G. F. Croft, G. Saphier, R. Leibel, R. Goland, H. Wichterle, C. E. Henderson, K. Eggan, Induced pluripotent stem cells generated from patients with ALS can be differentiated into motor neurons, Science 321 (2008) 1218-1221.

[35] I. H. Park, N. Arora, H. Huo, N. Maherali, T. Ahfeldt, A. Shimamura, M. W. Lensch, C. Cowan, K. Hochedlinger, G. Q. Daley, Disease-specific induced pluripotent stem cells, Cell 134 (2008) 877-886.

[36] Y. Asai, M. Tada, T. G. Otsuji, N. Nakatsuji, Combination of functional cardiomyocytes derived from human stem cells and a highly-efficient microelectrode array system: an ideal hybrid model assay for drug development, Curr. Stem Cell Res. Ther. (2010) epub ahead of print.

[37] K. A. D’Amour, A. G. Bang, S. Eliazer, O. G. Kelly, A. D. Agulnick, N. G. Smart, M. A. Moorman, E. Kroon, M. K. Carpenter, E. E. Baetge, Production of pancreatic hormone-expressing endocrine cells from human embryonic stem cells, Nat. Biotechnol. 24 (2006) 1392-1401. 

differentiation of hepatocytes from human embryonic stem cells exhibiting markers recapitulating liver development in vivo, Stem Cells 26 (2008) 894-902.

[39] K. Osafune, L. Caron, M. Borowiak, R. J. Martinez, C. S. Fitz-Gerald, Y. Sato, C. A. Cowan, K. R. Chien, D. A. Melton, Marked differences in differentiation propensity among human embryonic stem cell lines, Nat. Biotechnol. 26 (2008) 313-315.

[40] S. Chen, M. Borowiak, J. L. Fox, R. Maehr, K. Osafune, L. Davidow, K. Lam, L. F. Peng, S. L. Schreiber, L. L. Rubin, D. A. Melton, A small molecule that directs differentiation of human ESCs into the pancreatic lineage, Nat. Chem. Biol. 5 (2009) 258-265.

\section{Figure Legends}

\section{Fig. 1}

Multipotent progenitor cells in metanephric kidney. (A) In vitro colony formation from

a single progenitor cell within metanephric mesenchyme on feeder cells expressing Wnt4. (B) A single progenitor cell divides and differentiates into two renal lineages; (upper pannel) podocytes (red: peanut agglutinin; PNA) and proximal tubular epithelia 
(green: Lotus Tetragonobulus lectin; LTL), (lower) proximal (green: LTL) and distal tubular epithelia (red: E-cadherin, arrows). (C) Only cell population strongly expressing Sall1 (Sall1-GFP ${ }^{\text {high }}$ ) that contains multipotent progenitors (upper panels) differentiate into kidney structure in organ culture, whereas that weakly expressing Sall1 (Sall1-GFP low, lower) disappear. (D) Hematoxylin-eosin staining of sections of Sall1-GFP high aggregates at day 10. Tubule- (t) and glomerulus-like structures (g) are seen. Bars: (A, B) $50 \mu \mathrm{m}$, (C) $500 \mu \mathrm{m}$, (D) $25 \mu \mathrm{m}$.

\section{Fig. 2}

Pronephric kidney formed in vitro from undifferentiated cells in an amphibian. Double immunostaining of stage 42 equivalent explants (A, B) and a stage 40 Xenopus larvae (C, D) using pronephric tubule-specific antibody 3G8 (red) and pronephric duct-specific antibody 4A6 (blue). Whole-mount immunostaining patterns (A, C) and photomicrographs of sections (B, D) are shown. (A, B) Explants treated with activin A and retinoic acid contain both 3G8-positive (pronephric tubules, arrows) and 4A6-positive tubular structures (pronephric ducts, arrowheads). Bars: $1 \mathrm{~mm}$. 


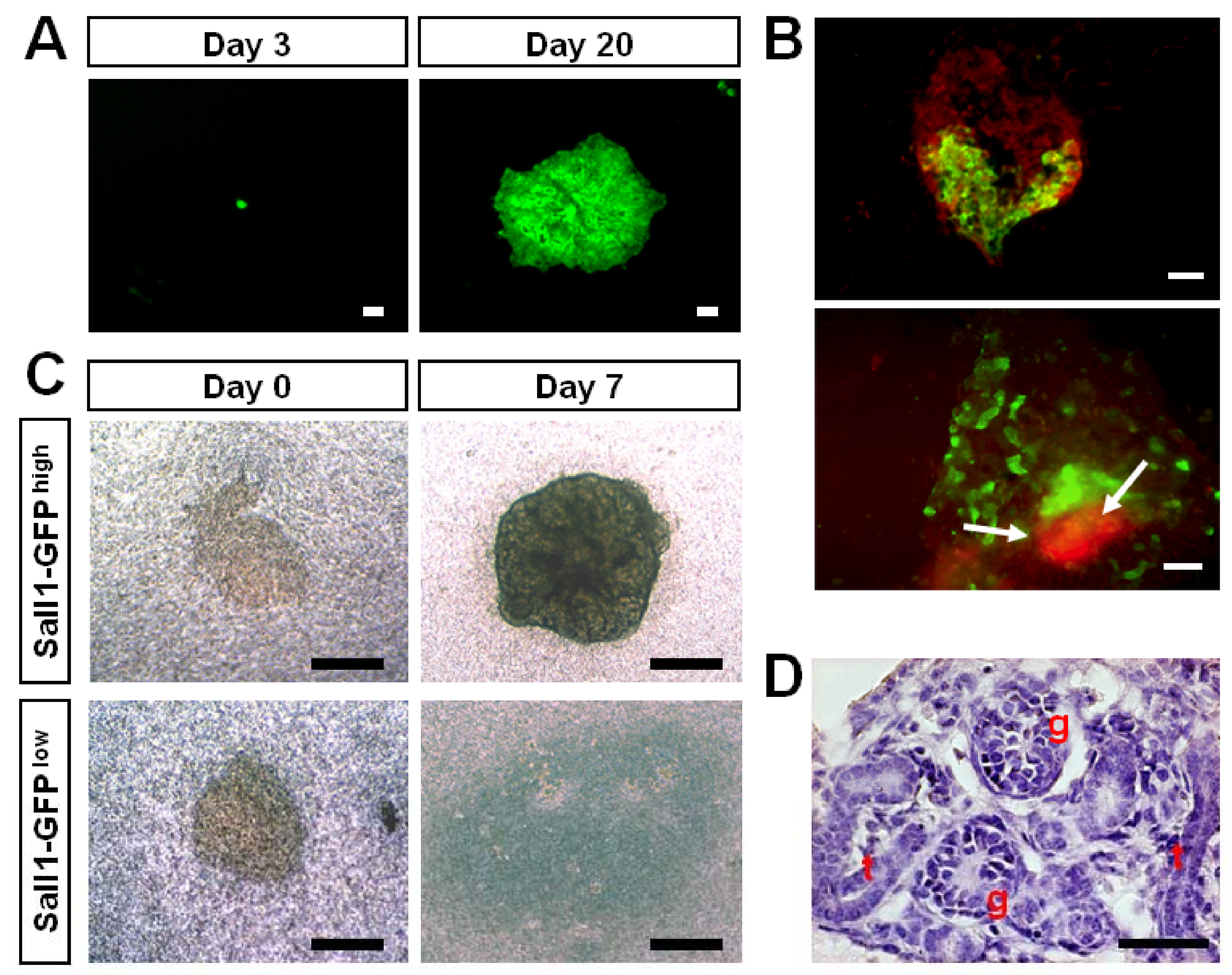



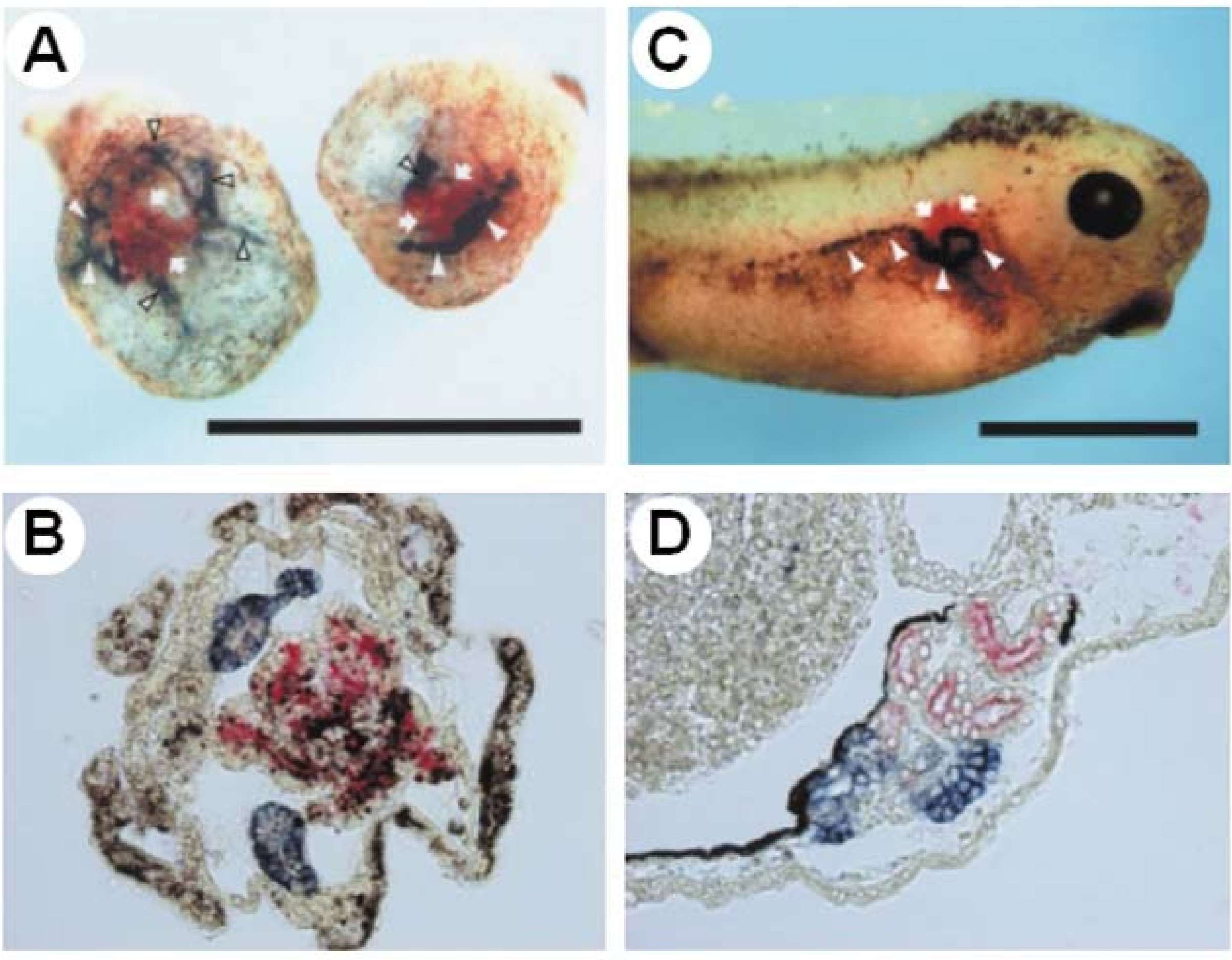